\title{
Über die fermentative Zersetzung der Thymonucleinsäure durch Schimmelpilze.
}

Von

Leonid Iwanoff.

(Aus dem botanischen Institut zu Leipzig.)

(Der Redaktion zugegangen am 25. Mai 1903.)

Die schon lange bekannte Beständigkeit der Nucleine gegen die Pepsinwirkung und das vorherrschende Vorhandensein dieser Stoffe in den an Formelementen reichen Zellkernen haben zu der Voraussetzung geführt, daß sie in die Zusammensetzung des lebendigen Gerüstes eingehen, in dessen Mitte die verschiedenen Zersetzungen verlaufen.

Solcher Ansicht gemäß unterliegen die Nucleinstoffe der Zersetzung in der lebenden Zelle nicht und zum Beispiel beim Hungern müssen sie relativ zunehmen.

Aber schon lange sind Tatsachen bekannt, welche gegen eine solche Vorstellung sprechen. Bei allen Versuchen mit Selbstverdauung ${ }^{1}$ ) bemerkten die Autoren das Erscheinen oder die Vermehrung der Nucleinbasen oder der anorganischen Phosphorsäure.

Die vitale Zersetzung der Nucleoproteide bei der Keimung der Samen beobachteten Salomon und Schulze, ${ }^{2}$ ) welche die Erscheinung der freien Nucleinbasen dabei feststellten.

Viele Beobachtungen in der Tierphysiologie beweisen, daß nucleinreiche Nahrung eine vermehrte Ausscheidung der Harnpurine zur Folge hat und daß wahrscheinlich eine Menge von Harnpurinen aus Prozessen stammen, die von der zugeführten Nahrung unabhängig sind. ${ }^{3}$ )

1) Die Literatur über Selbstverdauung der Hefe s. Kutscher, Diese Zeitschr., Bd. XXX, S. 59.

2) Salomon, Du Bois' Archiv 1881, S. 166, 361. Schulze und Barbieri, Journ. f. prakt. Ghem., N. F., Bd. 37, S. 358. Die Literatur über Vorkommen von freien Xanthinbasen in Pflanzen bei Schulze und Boßhard, Diese Zeitschr., Bd. IX, S. 437.

8) S.Burian u. Schur, Arch.f.d.ges. Phys., Bd.80, p.241, Bd.87, p.239. 
In vorigem Jahre ${ }^{1}$ ) verfolgte ich die Veränderungen des Eiweißphosphors bei der Keimung von Vicia und fand geradle gegen die verbreitete Ansicht, daß die Eiweißstoffe immer mehr und mehr ihren Phosphor verlieren und um dieses Element ärmer werden. ${ }^{2}$ )

Die schnell verlaufende Zersetzung zwang nicht nur einmal die verschiedenen Autoren, vorauszusetzen, daß der Organismus zu einer solchen Reaktion das Enzym benutzt.

Zuerst wurden natürlich die schon bekannten proteolytischen Enzyme: Pepsin, Trypsin, Erepsin untersucht.

Über die Verdaulichkeit der Nucleinsäure durch Trypsin finden wir Angaben in den Arbeiten von Popoff, Milroy, Umber, Araki. ${ }^{3}$ ) Der erste Forscher hat gefunden, daß das Thymusnuclein unter der Einwirkung des Trypsins sich auflöst, aber der Phosphor verbleibt in organischer Verbindung und anorganischer Phosphor erscheint nur in geringer Menge. Milroy zeigte an künstlichen und natürlichen Nucleinen, daß Trypsin einen Teil des Phosphors als Phosphorsäure abspaltet, aber daß deren Menge (von 6-47\% io des in Lösung über-

1) Berichte d. deutsch. bot. Gesellsch., Bd. 20, S. 366.

$\left.{ }^{2}\right)$ Lukian off, (Arch. des scienc. biolog. T. VI, p. 1, 2, T. VII, p. 168) beobachtete die Abnahme der Größe der Kerne beim Hungern um $40 \%$ und Nemser (Arch. d. scienc. biolog. T. VII, p. 221) zeigte, daß das Hungern die absolute Abnahme des Nuclein-P. in verschiedenen Tierorganen verursacht. S. auch A. Kossel, Diese Zeitschr., Bd. VII, S. 7.

Daß Zaleßki (Ber. d. deutsch. bot. Gesellsch., Bd. 20, S, 426.) keine Abnahme des Eiweißphosphors bei der Keimung des Lupinus angustifolius beobachtete, wird einfach damit erklärt, daß der Autor nur die letzten (vom 10. Tage) Stadien untersuchte, als der Stoffzerfall schon fast aufgehört hatte. (S. Merlis, Landw. Vers.-Stat., B. XVIII, S. 419.) Daß die Zersetzung während der versäumten Zeit sehr stark war, kann man aus der geringen Menge (10 mg. $\mathrm{P}_{2} \mathrm{O}_{5}$ auf 300 Pflanzen oder $12-15 \mathrm{~g}$ Trockensubstanz) der gebliebenen Eiweißphosphorsäure schließen. Da der Eiweißstickstoffgehalt $\mathrm{zu}$ dieser Zeit 1,5-2\% betrug, so kann man sicher sagen, daß die Eiweißstoffe der Axenorgane nur minimalste Mengen Nucleine (wahrscheinlich nur in den Knospen) enthalten.

s) Popoff, Diese Zeitschr., Bd. XIV, S. 524, Milroy, Diese Zeitschr., Bd. XXII, S. 307, Umber, Zeitschr. f. klin. Medizin, Bd. 43, 1901, S. 281, Araki, Diese Zeitschr., Bd. XXXVIII 1903, S. 84. 
gegangenen Gesamtphosphors) in verschiedenen Nucleinpräparaten sich verändert und die Menge überhaupt nicht groß ist.

Umber hat gefunden, daß das Pankreasnucleoproteid bis aauf verschwindende Reste sowohl von Pepsin wie von der Trypsinverdauung gelöst wird und daher die ältere Vorstellung, (daß die Kerneiweiße der Pepsinverdauung gegenüber resistent seien, beweiskräftige Widerlegung gefunden.')

Araki hat gezeigt, daß das Trypsin und das Erepsin die gelatinöse Nucleinsäure in eine lösliche Verbindung, ohne tief gu zersetzen, überführen können.

Auf solche Weise können sich die Nucleoproteide unter dem Einfluß des Ferments tierischen Ursprungs vollständig auflösen. Aber die Zerspaltung der Nucleinsäure ist zu unbedeutend, um im Pepsin oder Trypsin ein Ferment nicht nur für Eiweiß-, sondern auch für Nucleinzersetzung zu sehen.

Daher muß man voraussetzen, daß im Zersetzungsprozesse der Nucleinsäure ein sich solcher Reaktion speziell anpassendes Ferment teilnimmt.

$\mathrm{Zu}$ meinen Versuchen wählte ich die Schimmelpilze, welche besonders geeignet sind, verschiedene Fermente für die Zersetzung des ihnen gegebenen Substrats zu erzeugen. Die Nucleinsäure, welche ich für meine Versuche benutzte, war ganz genau nach Neumann²) bereitet. Ich habe aus $1 \mathrm{~kg}$ der präparierten Thymusdrüse $30 \mathrm{~g}$ des nucleinsauren Natrons ${ }^{3}$ ) von gelatinierender Modifikation a bekommen; das Präparat war so rein, daß weder Alkohol noch Essigsäure einen Niederschlag

1) Wenn einerseits die Nucleoproteide durch Pepsin vollständig gelöst werden können, so wissen wir jetzt andererseits, daß die phosphorfreien Eiweißstoffe, wie z. B. Zein (s. Szumowsky, Diese Zeitschr., B. XXXVI, S. 198), sehr schwer durch dieses angegriffen werden. Also die sogenannten «unverdauten Eiweißstoffe s stellen einen ganz zufälligen Komplex dar, welcher für die theoretische Physiologie kaum eine Bedeutung hat.

) Neumann, Arch. f. Anat. u. Physiol., physiol. Abt.: 1898, S. 374, 1899, Supplb. S. 552. Das Präparat habe ich in Heidelberg unter Leitung von Prof. Dr. Kossel bereitet, wofür ich ihm zu bestem Dank verpflichtet bin.

${ }^{3}$ ) In allen weiteren Versuchen diente dieses Salz, welches ich daher kurz als «Nucleinsäure» bezeichne.

Hoppe-Seyler's Zeitschrift f. physiol. Chemie. XXXIX. 
bildete. Auch konnte ich weder Biuretreaktion noch Millonsche Färbung konstatieren. Hingegen gab Salzsäure und "Witte»Pepton den voluminösen Niederschlag. Ammoniakalische Silberlösung und Magnesiamischung bildeten direkt keinen Niederschlag, wohl aber nach dem Sieden mit Säuren.

Das Abdampfen und Trocknen auf dem Wasserbade änderte die Eigenschaften der Nucleinsäure gar nicht, daher bewahrte ich sie in trockenem Zustand auf. Zunächst prüfte ich das so erhaltene Präparat, ob es mit Trypsin verdaut werden könnte.

Das Trypsin war nach Kühne aus dem sog. Trockenpankreas bereitet und seine Wirkungsfähigkeit war mit Fibrin geprüft. Dann wurden je $100 \mathrm{ccm}$ von dem nucleinsauren Natrium in zwei Erlenmeyerschen Kolben mit $0,5 \mathrm{~g} \mathrm{Na}_{2} \mathrm{CO}_{3}$ und $5 \mathrm{ccm}$ Trypsinlösung eingegossen. Ein Kolben stand $1 / 2$ Stunde auf dem siedenden Wasserbade und nachher wurden beide unter Zusatz von Thymol in den auf $37^{\circ}$ erhitzten Thermostaten gebracht. Nach 48 Stunden gaben beide Proben keine Reaktion mit ammoniakalischer Silberlösung und Magnesiamischung. Sogar die gelatinöse Konsistenz der Substanz erlitt keine Änderung, und die Fähigkeit, den Peptonniederschlag zu erzeugen, blieb unverändert. ${ }^{\text {) }}$

Wir müssen also feststellen, daß Trypsin keine Zersetzung der Nucleinsäure bis zum Auftreten der Phosphorsäure und der Xanthinbasen hervorruft.

Als Objekte für weitere Versuche dienen Aspergillus, Penicillium und Mucor. Ein Vorversuch mit der Kultur dieser Pilze auf der Nucleinsäure + Zucker zeigte, daß alle diese Pilze auf solchem Substrate wachsen können, und bei weiteren Versuchen benutzte ich nur Aspergillus niger und Penicillium glaucum.

Um zu prüfen, ob der Pilz die Nucleinsäure als Kohlenstoffquelle benutzen kann, wurden die Kulturen mit Nucleinsāure + Salze $\left(\mathrm{MgSO}_{4}\right.$ und $\left.\mathrm{KCl}\right)$ ohne andere Kohlenstoffquellen herge-

1) Araki hat den Übergang in eine lösliche Modifikation der Nucleinsäure nur bei längerer Trypsinwirkung beobachtet; s. oben. 
stellt.1) Die Pilze keimten auf solchem Substrat, bildeten nach einem Monat einige Flocken des versunkenen Mycelium, manchmal sogar mit kaum bemerkbaren seltenen Sporen, aber die Entwickelung war so gering, daß sie nur auf Kosten gewisser Beimischungen geschehen konnte.

In der Voraussetzung, daß für die Benutzung der Kohlenhydratgruppe des Nucleinsäuremoleküls zunächst diese zersetzen mußte, fügte ich, um den Anfang des Wachstums zu begünstigen, 0,2\% Glukose hinzu, aber die Entwickelung war auch in diesem Falle nicht besser.

Also muß man feststellen, daß die Nucleinsäure nicht als Kohlenstoffquelle dienen konnte.

Dasselbe Resultat wurde mit Pencillium erzielt.

In einer anderen Serie von Versuchen wurde zur Nucleinsäure Zucker hinzugefügt, welcher dann als Kohlenstoffquelle diente. Dann entwickelte sich der Pilz ganz gut, indem er den Stickstoff der Nucleinsäure benutzte.

Ich führe einige Versuche an:

\section{Versuch I.}

\section{Aspergillus niger.}

Die Kulturflüssigkeit hatte folgende Zusammensetzung:

$25 \mathrm{ccm}$ Nucleinsäure mit $57,6 \mathrm{mg} \mathrm{P}_{2} \mathrm{O}_{5}$ oder $25 \mathrm{mg} \mathrm{P}$ und $44 \mathrm{mg} \mathrm{N}$

$4 \mathrm{~g}$ Saccharose

$0,5 \mathrm{~g} \mathrm{MgSO}_{4}$

$0,1 \mathrm{~g} \mathrm{KGl}$

วัó $\mathrm{ccm} \mathrm{H}_{2} \mathrm{O}$

Nach 5 Tagen erhielt man stark entwickeltes meistenteils versunkenes Mycelium mit schwacher Sporenbildung. Das Gewicht des bei $90^{\circ}$ getrockneten Myceliums betrug $0,1430 \mathrm{~g}$ mit $10,78 \mathrm{mg}$ N. Die abfiltrierte Flüssigkeit reagierte sauer und für die Neutralisation verbrauchte sie ungefähr $15 \mathrm{ccm} 1 / 10 \mathrm{n}$-Natronlauge. Auf den Boden der Krystallisationsschale fiel der weiße Niederschlag der freien Nucleinsäure.

1) Die Kulturkolben wurden in allen Kulturen zunächst in dem Trockenschrank bei $150^{\circ}$ und dann 2 mal mit den Nährlösungen in dem Dampfsterilisator sterilisiert. 


\section{Versuch II.}

Aspergillus niger.

Die Kulturflüssigkeit hatte dieselbe Zusammensetzung wie im vorhergegangenen Versuche. Dauer der Kultur 6 Tage. Das Mycel war gut entwickelt, aber die Sporenbildung war gehemmt. Es wurde mit Wasser gewaschen, bei $90^{\circ}$ getrocknet und gewogen. Sein Gewicht betrug $0,107 \mathrm{~g}$ mit $11,76 \mathrm{mg}$ N. Die Filtratflüssigkeit reagierte stark sauer und auf dem Boden der Schale wurde ein weißer Niederschlag, die freie Nucleinsäure, abgesetzt. Die Menge der anorganischen Phosphorsäure (bei der direkten Fällung mit Magnesiamischung) in der Kulturflüssigkeit war gleich nur 11,2 $\mathrm{Mg}_{2} \mathrm{P}_{2} \mathrm{O}_{7}$.

\section{Versuch III.}

Penicillium glaucum.

Die Zusammensetzung der Kulturflüssigkeit war dieselbe wie in der vorhergehenden Kultur, nur der Zuckergehalt wurde bis $10 \mathrm{~g}$ vermehrt. Dauer der Kultur 10 Tage. Die Entwickelung war gut und die Sporenbildung ganz normal. Das mit 20!oiger $\mathrm{Na}_{2} \mathrm{CO}_{3}$ gewaschene und bei $90^{\circ}$ getrocknete Mycel wog $0,265 \mathrm{~g}$ und enthielt $11,6 \mathrm{mg} \mathrm{N}$.

Diese Versuche beweisen, da $\dot{\beta}$ die Schimmelpilze die Nucleinsäure als Stickstoffquelle benutzen können.

In weiteren Versuchen suchte ich die Zerspaltungsprodukte in der Filtrationsflüssigkeit aufzufinden und die ganze Bilanz der verbrauchten und unverbrauchten $\mathrm{N}$ - und P-Verbindungen $\mathrm{zu}$ bestimmen.

\section{Versuch IV.}

Aspergillus niger.

In die breite Schale mit $150 \mathrm{qcm}$ Oberfläche wurde eingegossen:

$$
\begin{aligned}
& 150 \mathrm{ccm} \text { von } 5 \% \text { Saccharose } \\
& \left.50 \mathrm{ccm} \mathrm{Nucleinsäure}{ }^{1}\right) \\
& 8 \mathrm{ccm} \mathrm{MgSO}_{4}(5 \%) \text { und } \\
& \text { einige Kryställchen von KCl. }
\end{aligned}
$$

1) Der N- und P-Gehalt war derselbe wie in den vorhergegangenen Versuchen. 
Außerdem wurde, weil man den schädlichen Einfluß der bei der Entwickelung gebildeten Säure (hauptsächlich Oxalsäure) auf die Sporenbildung bemerken konnte, zur Neutralisation dieser :Säure Kreide hinzugefügt.

Dauer der Kultur 32 Tage.

Temperatur $25^{\circ}$.

Die Entwicklung der Aspergillus war ganz normal mit guter Sporenbildung.

Das Mycel mit einer großen Menge von Krystallen des oxalsauren Kalks wurde abfiltriert, mit 2\% mit säurehaltigem Wasser gewaschen und auf zwei ungefähr gleiche Teile zerschnitten.

Die beiden bei $90^{\circ}$ getrockneten Teile wogen $2,0274 \mathrm{~g}$. Die eine Hälfte diente zur $\mathrm{N}$-Bestimmung, die andere zur $\mathrm{P}_{2} \mathrm{O}_{5}$ Bestimmung. Das schwachsaure ${ }^{1}$ ) Filtrat mit dem Waschwasser von $250 \mathrm{ccm}$ Volumen diente zur Bestimmung von Ammoniak (durch Destillation mit Magnesia), der Xanthinstoffe (mit ammoniakalischer Silberlösung) und der anorganischen Phosphorsäure.

Ich erhielt folgendes Resultat:

\begin{tabular}{c|c|c|c|c|c}
\hline \hline Es wurde . & $\begin{array}{c}\text { Gesamt- } \\
\mathbf{N .} \\
\mathbf{m g}\end{array}$ & $\begin{array}{c}\text { Ammoniak- } \\
\mathbf{N .} \\
\mathbf{m g}\end{array}$ & $\begin{array}{c}\text { Xanthin- } \\
\mathbf{N .} \\
\mathbf{m g}\end{array}$ & $\begin{array}{c}\text { Gesamt- } \\
\mathbf{P .} \\
\mathbf{m g}\end{array}$ & $\begin{array}{c}\text { Anorg. } \\
\mathbf{P .} \\
\mathbf{m g}\end{array}$ \\
\hline Gegeben . . . . & 87 & & & 50 & \\
Gefunden in Mycel & 58 & & & 14,56 & \\
Gefunden in Filtrat & & 14 & 7 & & 36,4
\end{tabular}

Also aus den in Form der Nucleinsäure vorhandenen $87 \mathrm{mg} \mathrm{N}$ wurden $58 \mathrm{mg}$ oder 66\% zum Bau des Mycels verbraucht, 24\% wurden als unverbrauchte Ammoniaksalze und Xanthinstoffe und die übriggebliebenen 10 Prozent als unbestimmte unverbrauchte Zersetzungsprodukte gefunden.

Die Verhältnisse in der P-Bilanz sind andere. Zum Bau des Mycels wurden nur 30\% verbraucht und 70\% als abgespaltene Phosphorsäure waren ganz unberührt geblieben.

1) Die Kreide wurde ganz verbraucht. 
Wir können feststellen, daß die Zersetzung der Nucleimsäure eine vollständige war, weil die ganze Menge der in deer Nucleinsäure gebundenen Phosphorsäure durch den Pilz albgespalten wurde.

\section{Versuch V.}

Unter ganz denselben Bedingungen wie bei dem vorhergegangenen Versuche wurde Penicillium glaucum kultiviert. Nach 7 Tagen wurde das gut entwickelte, sporentragende Mlycelium mit Soda und Wasser gewaschen, getrocknet und wog $0,785 \mathrm{~g}$. Es enthielt $34,5 \mathrm{mg} \mathrm{N}$ und $6,4 \mathrm{mg} \mathrm{P}$. In demselbren Filtrat wurden $12,1 \mathrm{mg}$ von anorganischem Phosphor und $7 \mathrm{mg} \mathrm{N}$ der Xanthinstoffe gefunden.

\begin{tabular}{c|c|c|c|c}
\hline Es wurde & $\begin{array}{c}\text { Gesamt- } \\
\mathrm{N} . \\
\mathrm{mg}\end{array}$ & $\begin{array}{c}\text { Xanthin- } \\
\mathrm{N} . \\
\mathrm{mg}\end{array}$ & $\begin{array}{c}\text { Gesamt- } \\
\mathrm{P.} \\
\mathrm{mg}\end{array}$ & $\begin{array}{c}\text { Anorgan. } \\
\mathrm{P.} \\
\mathrm{mg}\end{array}$ \\
\hline Gegeben . . . . . & 87 & & 50 & \\
Gefunden in Mycel . & 34,5 & & 6,4 & \\
Gefunden in Filtrat . & & 7 & & 12,1
\end{tabular}

Auch hier fand wie beim Aspergillus eine tiefe Zersetzung der Nucleinsäure statt, aber nur der'kleinere Teil (weniger als die Hälfte), aus organischer Phosphorsäure berechnet, wurde wegen der kurzen Zeit zersetzt.

Diese Versuche beweisen, daß die Pilze die Nucleinsäure sehr tief zersetzen können.

Jetzt kommt die Frage, mit welchen Mitteln diese ein solches Resultat erreichen?

Am einfachsten ist es, vorauszusetzen, daß die in großer Menge durch die Pilze erzeugte Oxalsäure eine Rolle dabei spielt. Wir wissen, daß die freie Nucleinsäure viel weniger beständig ist als ihre Salze. Es war möglich, daß die durch Oxalsäure ausgeschiedene Nucleinsäure unter der Wirkung der ersteren zersetzt wurde.

Um diese Voraussetzung zu prüfen, wurden die folgenden Versuche angestellt. 


\section{Versuch VI.}

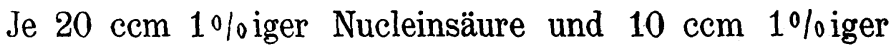
Lösung der Oxalsäure wurden in einen Erlenmeyerschen Kolben eingegossen und der letztere bei $33^{\circ}$ aufgestellt.

Nach 9 Tagen zeigte die mit Ammoniak neutralisierte Flüssigkeit keinen Niederschlag weder durch Magnesiamischung noch Silberlösung.

\section{Versuch VII.}

Es wurde genommen: $25 \mathrm{ccm}$ der $0,75 \%$ igen Nucleinsäure mit $15 \mathrm{mg} \mathrm{P}_{2} \mathrm{O}_{5}, 1,5 \mathrm{~g}$ der Oxalsäure und $50 \mathrm{~cm} \mathrm{H}_{2} \mathrm{O}$.

Temperatur $33^{\circ}$.

Zunächst wurde der weiße Niederschlag der freien Nucleinsäure ausgeschieden, aber am nächsten Tage hatte er sich wieder gelöst. Nach 7 Tagen zeigte die mit Ammoniak neutralisierte Flüssigkeit keinen Niederschlag weder durch Magnesiamischung noch Silberlösung.

Diese 2 Versuche sprechen unzweideutig gegen die Voraussetzung, daß die Oxalsäure die Nucleinsäure tief zersetzen kann, aber nicht gegen die Voraussetzung, daß diese die leichtere Abspaltung der Xanthinstoffe verursachen kann.

Die Mißerfolge der Reaktion mit Silberlösung konnten von der hemmenden Wirkung der noch nicht zersetzten Nucleinoder der .Thyminsäure abhängen. $\left.{ }^{1}\right)$ Der nächste Versuch bestätigte diese Voraussetzung nicht.

\section{Versuch VIII.}

In 2 Erlenmeyersche Kolben A und B wurden je $50 \mathrm{ccm}$ der $1 \%$ igen Nucleinsäure und in den Kolben $A$ außerdem noch $50 \mathrm{ccm}$ Oxalsäure $(5 \%)$ eingegossen.

$\mathrm{Zu}$ beiden wurde die genügende Menge Chloroform hinzugefügt und beide wurden bei $31^{\circ}$ stehen gelassen. Schon beim Eingießen der Oxalsäure fiel ein weißer Niederschlag von Nucleinsäure, welcher am nächsten Tage verschwand.

Nach 3 Tagen wurde das Chloroform auf dem Wasser-

1) S. Kossel und Neumann, Diese Zeitschr., B. XXII, S. 74. 
bade abgedunstet und der Inhalt der Kolben mit Magnesiamischung und Silberlösung geprüft. Die Prüfung gab eĩn negatives Resultat. Dann setzte ich zu den Flüssigkeiten kalt gesättigtes Barytwasser bis zur schwach alkalischen Reaktion hinzu und lieb es bis zum nächsten Tage stehen. Der Inhalt des Kolben A gab reichen Niederschlag, der des Kolbens B nur leichte Trübung. Der von dem Baryt mit Schwefelsäure befreite und gelöste Niederschlag gab keine Reaktion auf Guanin (Xanthinfärbung und Guaninpicrat). Das Filtrat wurde in die $1 \frac{1}{2}$ fache Menge Alkohol mit einigen Tropfen von Chlorbaryum hineingegossen. Die von dem abgeschiedenen Niederschlage abgegossene Flüssigkeit gab auch keine zweifellose Adeninreaktion (mit Goldchlorid) auf.

Also können wir feststellen, daß die Oxalsäure keine tiefe Zersetzung ${ }^{1}$ ) erzeugen kann und ein anderer zersetzender Faktor gesucht werden muß. Hierfür wurden die folgenden Versuche angestellt:

\section{Versuch IX.}

Das sporentragende Mycel von einer einmonatlichen Kultur (siehe Versuch IV) wurde gewaschen, bei $30^{\circ}$ getrocknet, dann im Mörser mit etwas Wasser und Kieselguhr zerrieben. Die abfiltrierte Flüssigkeit $(18 \mathrm{ccm})$ wurde in zwei gleiche Teile geteilt und beide in Erlenmeyersche Kolben A und B eingegossen und das zerriebene Mycel selbst wurde in einen dritten Kolben $\mathrm{G}$ eingebracht. Dann stand der Kolben B ungefähr 15 Minuten auf dem siedenden Wasserbade und zu allen dreien wurde je $28 \mathrm{ccm}$ der Nucleinsäure (mit $25 \mathrm{mg} \mathrm{P}_{2} \mathrm{O}_{5}$ ) und der Überschuß des Chloroforms hinzugefügt.

Nach zehntägigem Stehen bei $33^{\circ}$ wurde das Chloroform auf dem Wasserbade abgedunstet und der Inhalt jedes Kolbens abfiltriert.

Die Magnesiamischung gab einen Niederschlag, welcher nach dem Glühen wog:

1) Die in allen Versuchen beobachtete Auflösung der zunächst ausgeschiedenen Nucleinsäure zeigt, daß Oxalsäure irgendwelche Änderungen in der Nucleinsäure verursacht. 


$$
\begin{aligned}
& \text { In dem Kolben } \mathrm{A} \quad 7 \mathrm{mg} \text { oder } 4,48 \mathrm{mg} \mathrm{P}_{2} \mathrm{O}_{5} \text {. } \\
& \text { « \& B Spuren } \\
& \text { « « C } 23 \mathrm{mg} \text { oder 14,72 mg } \mathrm{P}_{2} \mathrm{O}_{5} \text {. }
\end{aligned}
$$

\section{Versuch X.}

Das Mycel, welches auf der Nucleinsäure (25 ccm) mit $4 \mathrm{~g}$ Dextrose und Kreide (5 g) gezogen wurde, wurde noch vor der Sporenbildung genommen, mit Wasser gewaschen und im Mörser mit Kieselguhr zerrieben. Die breiartige sauer reagierende Masse wurde in zwei gleiche Teile (je $8 \mathrm{ccm}$ ) geteilt und in den Kolben A und B hineingebracht. Der Kolben A stand 15 Minuten auf dem Wasserbade und dann wurde zu beiden je $25 \mathrm{ccm} 4 \%$ iger Nucleinsäure (mit $150 \mathrm{mg} \mathrm{P}_{2} \mathrm{O}_{\overline{5}}$ ) und ein Überschuß von Chloroform hinzugefügt.

Nach 2 Wochen Stehens bei $33^{\circ}$ wurde das Chloroform abgedunstet, der Inhalt wurde abfiltriert. Die Magnesiamischung erzeugte einen reichlichen Niederschlag in dem Kolben B und Spuren davon in dem Kolben A. Der geglühte Niederschlag wog:

$$
\begin{array}{lrl}
\text { in Kolben A } & 5,0 \mathrm{mg} \text { oder } 3,2 \mathrm{mg} \mathrm{P}_{8} \mathrm{O}_{5} \\
\text { in Kolben } \mathrm{B} & 125,1 & \rightarrow 7,0 \gg \mathrm{P}_{8} \mathrm{O}_{5} \text {. }
\end{array}
$$

Dann wurden die von dem mit Magnesiamischung erzeugten Niederschlage abfiltrierten Flüssigkeiten mit der ammoniakalischen Silberlösung gefällt.

Der nur in dem Kolben B ausgeschiedene Niederschlag der Xanthinstoffe wurde nach eintägigem Stehenlassen abfiltriert, mit Wasser gewaschen, in die Kjeldahlschen Kolben hineingebracht und darin zunächst eine halbe Stunde mit Magnesia gekocht und schließlich nach Kjeldahl verbrannt. Die Menge des Xanthinbasenstickstoffs betrug 12,7 mg.

Diese Versuche zeigen, daß

1. die tiefe Zersetzung bis zum Auftreten der Phosphorsäure und der Xanthinbasen auch nach dem Tode fortdauert und

2. daß die Wirkung des zersetzenden Agens durch hohe Temperatur ganz gehemmt wird.

Folglich kommen wir zum Schluß, daß diese Zersetzung durch das in den Pilzzellen erzeugte Enzym verursacht wird. 
$\mathrm{Ob}$ dieses Enzym in die umgebende Flüssigkeit durch die lebende Pilzzelle ausgeschieden wird, ist sehr schwer zu entscheiden. Die in der vom Mycel abfiltrierten Flüssigkeit beobachtete Zersetzung (Versuch IX) konnte durch das Ferment verursacht werden, welches aus den nie fehlenden toten Zellen in die umgebende Flüssigkeit diffundierte.

Um zu beweisen, daß das nucleinzersetzende Enzym mit dem proteolytischen Enzym nicht identisch ist, wurden die folgenden Versuche angestellt:

\section{Versuch XI.}

Die kapillaren Glasröhrchen wurden nach dem Mettschen Verfahren ${ }^{1}$ ) durch die thymolhaltige, mit fein verteilter Tusche gefärbte Gelatine gefüllt. Ebensolche Röhrchen wurden auf dieselbe Weise mit gelatinierender Nucleinsäure gefüllt. Je ein Paar von Röhrchen mit Gelatine und Nucleinsäure wurden in die Probierröhrchen mit derjenigen Flüssigkeit eingelegt, welche von dem auf der Nucleinsäure mit Zucker ausgewachsenen Mycel abfiltriert wurde.

Zur Kontrolle wurden dieselben Röhrchen in dieselbe, aber vorher abgekochte Flüssigkeit eingebracht.

Temperatur des Versuches war $18^{\circ}$. Nach 48 Stunden waren die beiden mit der Nucleinsäure gefüllten Kapillaren ganz leer, die mit Gelatine gefüllten und die Kontrollröhrchen waren ganz voll.

\section{Versuch XII.}

Der vorhergegangene Versuch wurde noch einmal mit derselben Flüssigkeit wiederholt. Wieder wurde nach 48 Stunden die vollständige Auflösung der Nucleinsäure und das Intaktbleiben der Gelatine- und der Kontrollröhrchen festgestellt. ${ }^{2}$ ) Die Gelatineröhrchen blieben sogar noch 10 Tage ganz intakt.

1) Siehe Hoppe-Seyler's Handbuch der phys. und pathol. Analyse, 1903, S. 382. Siehe auch Butkewitsch, Jahrbüch. für wiss. Botan., Bd. 38, S. 148.

2) Ich muß hier bemerken, daß ich wegen der Löslichkeit der Nucleinsäure immer die Auflösung dieser in den Kontrollröhrchen am dritten Tage beobachtete. Daß es sich hier nur um Auf lösung (nicht um Zersetzung) handelt, haben wir schon früher gesehen. 
Diese Versuche zeigen, daß das nucleinspaltende Enzym mit dem proteolytischen Enzym sehr wahrscheinlich nicht identisch ist und daher den besonderen Namen: "Nuclease» verdient. ${ }^{1}$ )

Was die Bedeutung dieses neuen Enzyms anbetrifft, kann man sagen, daß diese weit über den hier beschriebenen Fall hinausreicht.

Jede Zelle im embryonalen Zustand besitzt eine große Menge der Nucleoproteide, ${ }^{2}$ ) welche bei der Entwickelung zersetzt werden. Aus den Untersuchungen Umbers (siehe oben) wissen wir, daß das Eiweiß der Nucleoproteide durch Pepsin oder Trypsin verdaut werden kann.

Die eben beschriebenen Tatsachen sprechen dafür, dab der andere Komponent der Nucleoproteide, die Nucleinsäure, auch einer Zersetzung mittels eines speziellen Enzyms, Nuclease, unterliegt.

Also ist es sehr wahrscheinlich, daß die Nucleoproteide durch die embryonalen Zellen aufgespeichert werden, damit sie bei weiterer Entwickelung durch Nuclease zersetzt werden.

Leipzig.

Botanisches Institut.

1) Emmerich und Löw (Zeitschr. f. Hygiene, Bd. 36, S. 9) nennen Nucleasen die aproteolytischen Enzyme einiger pathogenen Bakterien, welche das Protoplasma dieser Bakterien auflösen können, ‘weil sie die Nucleoproteide des Bakterienprotoplasmas auflösen». Aber wir wissen, daß das Auflösen der Nucleoproteide ohne weiteres durch Pepsin und Trypsin erzeugt werden kann. Nach der Terminologie von Duclaux kann man nur ein solches Ferment Nuclease nennen, welches die Nucleinsäure zersetzen kann.

2) Der Weizenembryo z. B. enthält nach Osborne $3,5 \%$ der Nucleinsäure (Diese Zeitschr., Bd. XXXVI, S. 85). 